FORMATION Formation emploi

Revue française de sciences sociales

131 | Juillet-Septembre 2015

Le Bac Pro a 30 ans

\title{
Apprendre un métier ou poursuivre ses études? Les élèves de lycée professionnel face à la réforme du bac pro trois ans
}

To learn a job or to continue studies in the higher education? the pupils of professional high school in the face of the reform of the vocational high school diploma three years

Handwerk lernen oder Studium fortsetzen? Fachgymnasiasten und die Reform des Fachabiturs (Reduzierung auf 3 Jahre Unterricht)

Aprender un oficio o seguir estudiando? Los alumnos de escuelas técnicas frente a la reforma del bachillerato técnico de tres años

Aziz Jellab

\section{OpenEdition}

Journals

Édition électronique

URL : http://journals.openedition.org/formationemploi/4484

DOI : 10.4000/formationemploi.4484

ISSN : 2107-0946

Éditeur

La Documentation française

Édition imprimée

Date de publication : 15 octobre 2015

Pagination : 79-99

ISSN : 0759-6340

\section{Référence électronique}

Aziz Jellab, «Apprendre un métier ou poursuivre ses études ? Les élèves de lycée professionnel face à la réforme du bac pro trois ans », Formation emploi [En ligne], 131 | Juillet-Septembre 2015, mis en ligne le 15 octobre 2017, consulté le 30 octobre 2020. URL : http://journals.openedition.org/ formationemploi/4484; DOI : https://doi.org/10.4000/formationemploi.4484 


\title{
Apprendre un métier ou poursuivre ses études? \\ Les élèves de lycée professionnel face à la réforme du bac pro trois ans
}

\begin{abstract}
AzIZ JELLAB
Docteur en sociologie et en sciences de l'éducation et habilité à diriger des recherches. Inspecteur général de l'Education nationale et chercheur associé au CERIES

(Centre de recherche Individus, Epreuves, Sociétés), université Lille 3
\end{abstract}

Résumé

\begin{abstract}
Apprendre un métier ou poursuivre ses études ? Les élèves de lycée professionnel face à la réforme du bac pro trois ans
\end{abstract}

Créé pour élever les niveaux de qualification et répondre aux besoins en main-d'œuvre dans certains secteurs professionnels, le baccalauréat professionnel permet de plus en plus la poursuite d'études dans l'enseignement supérieur. En mobilisant une enquête de terrain qualitative menée auprès d'élèves préparant un baccalauréat professionnel ou un BTS (brevet de technicien supérieur), l'article vise à mettre en perspective les mutations induites par le bac pro sur le rapport aux savoirs et l'engagement dans des études supérieures. Si le baccalauréat professionnel contribue à l'ascension scolaire d'une partie des diplômés, il risque de perdre de sa légitimité professionnelle à mesure que progresse la part des bacheliers professionnels parmi les étudiants.

Mots clés : enseignement technique-professionnel, baccalauréat professionnel, politique de l'éducation, cheminement scolaire, poursuite d'études, savoir, étude de cas

Abstract

To learn a job or to continue studies in the higher education? The pupils of professional high school in the face of the reform of the vocational high school diploma three years

Created to bring up the levels of qualification and meet the needs in workforce in certain professional domains, the vocational high school diploma allows more and more the continuation of studies in the higher education. By mobilizing a qualitative survey of ground led with pupils preparing a vocational high school diploma or a BTS (two years technical degree), the article aims at putting in perspective the evolutions inferred by the vocational high school diploma on the relationship in the knowledges and the commitment in higher education. If the vocational high school diploma parti- 
cipates in the school ascent of a part of the graduates, it risks to lose of its professional legitimacy as progresses the part of the professional holders of high school diploma among the students.

Keywords: technical \& vocational education, vocational baccalaureate, education policy, school paths, continuation of education, knowledge, case study

Journal of Economic Literature: I 21, J 24

Traduction : Auteur

En France, le lycée professionnel (LP) accueille près de 700000 élèves, soit le tiers des publics du second cycle de l'enseignement secondaire. Après la chute de ses effectifs, dans les années 70 et 80 , au profit des filières technologiques de lycée, le LP a enregistré une inversion de cette tendance, surtout au lendemain de la généralisation du baccalauréat professionnel préparé en trois ans. Désormais, le parcours des élèves est aligné symboliquement sur celui des élèves qui préparent un baccalauréat général ou technologique puisqu'ils deviennent bacheliers professionnels au bout de trois ans d'études après le collège au lieu de quatre auparavant. La réforme du baccalauréat professionnel a conduit, d'une part, à la recomposition des publics accueillis, les élèves entrant en LP étant légèrement plus jeunes que les générations précédentes ; d'autre part, au renforcement de la polarisation scolaire des deux diplômes préparés, désormais emblématiques, à savoir le baccalauréat professionnel et le CAP (certificat d'aptitude professionnelle).

En participant de l'élévation des niveaux de qualification, le baccalauréat professionnel contribue aussi au mouvement de massification de l'enseignement supérieur, notamment au sein des STS (sections de technicien supérieur) et des universités.

Mais l'engouement pour l'enseignement supérieur procède également de l'interaction entre une biographie scolaire et sociale, du rapport aux savoirs sur fond de confrontation à des activités scolaires et professionnelles - y compris lors des périodes de stages en milieu professionnel - et à des pratiques pédagogiques spécifiques (Jellab, 2005, a, b). Quels effets de la réforme du bac pro sur le rapport aux savoirs chez les élèves de LP peut-on mettre en évidence ? En mobilisant un matériau issu d'une enquête qualitative menée auprès d'une vingtaine d'élèves, notre propos ici vise à montrer comment leur expérience contribue à forger le projet de poursuivre des études dans l'enseignement supérieur court. Cette tendance pose pour le moins la question du statut du baccalauréat professionnel et de ses finalités. Elle permet aussi de réinterroger les analyses sociologiques dont on dispose, notamment celles qui voient dans la poursuite des études la manifestation d'une " revanche " vis-à-vis de la voie générale quand, pour notre part, nous y percevons l'effet logique d'une nouvelle manière de s'approprier son expérience et de s'inscrire dans un processus d'émancipation scolaire et sociale. 


\section{Quand la réforme du baccalauréat professionnel en trois ans rencontre de nouvelles aspirations scolaires}

La réforme dite " bac pro 3 ans " a eu des effets pluriels dont il est difficile de mesurer l'ampleur, faute de recul historique nécessaire. Néanmoins, elle a contribué à améliorer l'attractivité des études en LP ${ }^{1}$. Il convient d'être prudent avec la notion de choix de la voie professionnelle qui couvre de nombreuses ambiguïtés et cache le plus souvent un processus de rationalisation de son devenir scolaire ; cependant, il faut également en nuancer les effets sur le rapport aux études et aux savoirs. En effet, c'est la confrontation à des activités scolaires et professionnelles qui concourt à la propension à poursuivre des études; dès lors, elle dessine un univers du possible en faisant émerger des projets d'avenir plus ou moins élaborés ( $c f$. tableau 1). La progression des effectifs d'élèves poursuivant leurs études dans l'enseignement supérieur interroge le rôle du baccalauréat professionnel et, par extension, celui du LP, eu égard au marché du travail, à l'entrée dans la vie active. Elle dessine différentes finalités au diplôme, finalités qu'il faut distinguer selon qu'il s'agit de l'institution scolaire, des branches professionnelles ou des élèves.

\subsection{Pour de nombreux élèves de lycée professionnel, le bac pro 3 ans n'est plus une fin en soi}

Le processus de scolarisation ayant conduit à une forte élévation des niveaux de qualification, en France, a suscité un engouement pour les études longues chez les nouvelles générations ; cet engouement procède aussi des difficultés d'insertion sur le marché du travail. Mais l'évitement de la voie professionnelle tenait aussi à la forte corrélation entre ce champ de formation et l'occupation de statuts professionnels subordonnés et précaires. La donne change sensiblement dès lors que la voie professionnelle devient propédeutique à la poursuite d'études. Alors que nombre d'élèves continuent à vivre leur entrée en LP comme une chute scolaire renforcée par l'inégal prestige entre la voie professionnelle et la voie générale et technologique (Jellab, 2001 ; 2009), la réforme du bac pro 3 ans a pu attirer une partie des collégiens ; leur âge - on y compte en effet moins de redoublants par rapport aux générations antérieures - leur permettant de se projeter sur des études plus longues. Convoiter un diplôme du supérieur qui assure davantage de chances de se stabiliser professionnellement compte parfois tout autant que la quête d'une identité sociale rapprochant des élèves, issus majoritairement de

1. Une enquête par questionnaire, conduite en Loire-Atlantique par P-Y. Bernard et V. Troger (2011) auprès d'élèves, révèle qu'une majorité d'entre eux - $71 \%$ - affirme avoir choisi en premier vœu le LP. $60 \%$ des élèves interrogés envisagent de poursuivre des études en STS, ce qui confirmerait le rôle propédeutique du baccalauréat professionnel pour l'accès à l'enseignement supérieur court. Il faudra observer si cette tendance se confirme et s'il n'existe pas des effets spécifiquement contextuels : maintien d'une tradition industrielle et ouvrière locale, une dynamique marché du travail favorable à l'emploi, notamment. 
milieu populaire, des étudiants ; le recrutement social de ces derniers étant en effet dans l'ensemble plus favorisé. Le Décret N²005-1037 du 26 Août 2005 stipule que l'admission dans une section de technicien supérieur est de droit pour les élèves et les apprentis obtenant la mention bien ou très bien au baccalauréat professionnel. Cette disposition institutionnelle s'est doublée d'autres critères de recrutement, les académies étant sommées de réserver des places en STS pour les nouveaux bacheliers professionnels; elle a marqué un tournant qui inscrit le baccalauréat professionnel dans une nouvelle dynamique de l'offre et de la demande, suscitant un engouement pour la poursuite des études qui s'est, depuis, confirmé.

Ainsi, près de $25 \%$ des titulaires du baccalauréat professionnel entament des études dans l'enseignement supérieur court et à l'université.

\section{Tableau 1 : Evolution des taux d'inscription des bacheliers professionnels dans les différentes filières de l'enseignement supérieur}

\begin{tabular}{|l|c|c|c|c|c|}
\hline & $\mathbf{2 0 0 0}$ & $\mathbf{2 0 0 3}$ & $\mathbf{2 0 0 6}$ & $\mathbf{2 0 0 9}$ & $\mathbf{2 0 1 0}$ \\
\hline $\begin{array}{l}\text { Université hors } \\
\text { IUT }\end{array}$ & 6,4 & 6,3 & 5,8 & 6,9 & 6,9 \\
\hline STS & 9,7 & 14,4 & 15,5 & 17,7 & 18,4 \\
\hline IUT & 0,5 & 0,7 & 0,7 & 0,8 & 0,8 \\
\hline CPGE & 0,0 & 0,0 & 0,0 & 0,0 & 0,0 \\
\hline Total & 16,6 & 21,4 & 22 & 25,4 & 26,1 \\
\hline
\end{tabular}

Sigles : STS : sections de technicien supérieur ; IUT : Institut universitaire de technologie ; CPGE : Classe préparatoire aux grandes écoles. Source : Direction de l'évaluation, de la prospective et de la performance, l'Etat de l'école, 2012, p. 47.

Cette poursuite d'études ne s'opère pas sans embûches car bien que la réussite des bacheliers professionnels soit supérieure en BTS, comparée à celle constatée à l'université, l'échec touche une part importante des étudiants. ${ }^{2}$ En 2013, dans le domaine de la production, qui enregistre le plus fort taux de progression des bacheliers professionnels, $68 \%$ ont obtenu leur BTS, et c'était le cas de $55 \%$ dans le domaine des services ${ }^{3}$. Le taux de réussite chute nettement chez les bacheliers poursuivant leurs études à l'université. Ainsi, le suivi sur trois et quatre années d'étudiants issus de la cohorte 2008 montre un taux de réussite, dans le cursus licence, de l'ordre de 3,2 \% en trois ans, de $2,5 \%$ en quatre ans, alors que le taux de réussite global, tous baccalauréats confondus, est de respectivement 27,3 et $12,4 \%$.

2. Comme le souligne Sylvaine Péan, "Au final, si l'on tient compte des abandons en cours d'études et que l'on rapporte les diplômés de STS aux inscrits de première année, le taux de réussite des bacheliers professionnels serait de l'ordre de 45\% ", in Les bacheliers professionnels dans l'enseignement supérieur, Note d'information, MEN, juin 2012-04, p. 7.

3. Repères et références statistiques, ministère de l'Education nationale, 2014. 
Le choix d'entamer des études supérieures ne peut être apprécié sociologiquement que si l'on prête attention au rapport aux savoirs chez les élèves et à ce qui, dans les apprentissages effectués, fait - ou non- sens pour eux, s'inscrit plus ou moins dans un projet d'études, d'insertion, etc. En effet, le statut dominé du lycée professionnel, au sein de l'institution scolaire, et l'image industrielle associée à un ordre d'enseignement qui scolarise davantage d'élèves dans les spécialités des services n'empêchent pas la grande diversité des expériences et des manières de se mobiliser ou non sur les savoirs. Cela nous amène, d'une part, à nuancer les analyses et les interprétations proposées par d'autres travaux (Beaud, Pialoux, 2003) et, d'autre part, à reconsidérer l'hypothèse selon laquelle la poursuite d'études dans l'enseignement supérieur ne serait que l'expression d'une " revanche " sur la " voie normale " que constitue le baccalauréat général. En effet, les observations que nous avons menées, et que nous développerons plus loin, tout en mettant en évidence des tensions et une critique récurrente à l'égard du collège et du système scolaire en général, font apparaître que de nombreux élèves de LP vivent positivement leur expérience ; expérience que l'on ne saurait réduire à une rationalisation d'un destin entériné.

\subsection{Le projet de poursuivre des études à l'épreuve du rapport aux savoirs}

Le rapport aux savoirs en lycée professionnel s'élabore à partir d'une confrontation avec des activités scolaires et professionnelles ; cette confrontation s'inscrit aussi dans des interactions pédagogiques spécifiques et des relations avec les pairs. Et lorsque l'on interroge les élèves sur ce qu'ils pensent apprendre en LP, ils évoquent en premier lieu les domaines professionnels, ce qui ne signifie pas qu'ils y adhèrent subjectivement. En outre, le point de vue sur leur scolarité reste fortement structuré par la filière et la spécialité de formation (Jellab, 2003).

Les différentes formes de rapport aux savoirs observées constituent une grille de lecture (cf. encadré 1) permettant de rendre compte de la diversité des manières d'étudier qui, elle, ne procède pas de la seule origine sociale, majoritairement populaire, des élèves de LP, mais se construit aussi au rythme des interactions pédagogiques, de l'emprise plus ou moins effective sur les objets d'apprentissage et de la capacité à s'y appuyer pour élaborer des projets d'avenir. Etre élève au LP, c'est se confronter à des savoirs, à des pratiques pédagogiques et construire des projets d'avenir. Porter le projet parental de "devenir ouvrier ", " grandir en apprenant un métier » ou espérer " aller plus loin dans les études ", constituent des buts hétérogènes dont la genèse est biographique (ou socio-subjective) mais aussi scolaire. C'est à l'articulation entre cette histoire biographique, les savoirs enseignés en LP, les rapports de sociabilité construits au sein et en dehors de celui-ci et les pratiques pédagogiques - subsumées par le regard porté par les enseignants sur leur public -, que l'on peut saisir la diversité de l'expérience des élèves.

Sans que l'on puisse véritablement apprécier ce qui, dans la propension à la poursuite d'études revient à la réforme du bac pro 3 ans et ce qui relève du rapport aux savoirs, nous postulons que c'est bien dans l'interaction entre ces deux dimensions que prend sens 
le projet de prolonger le temps des études. L'enquête menée auprès d'élèves préparant un bac professionnel ou un BTS livre quelques enseignements permettant d'apprécier la rencontre entre une évolution institutionnelle et l'émergence de projets personnels dans lesquels la poursuite d'études dessine de nouveaux horizons, l'insertion professionnelle n'étant qu'un objectif parmi d'autres.

\section{Encadré 1 : La diversité des formes de rapport aux savoirs chez les élèves de lycée professionnel}

Conceptualisée sous un angle sociologique par Bernard Charlot (1987; 1997), la notion de « rapport $a u(x)$ savoir(s) » s'inscrit dans un regard constructiviste, pour lequel les apprentissages scolaires n'ont de sens qu'en référence à des apprentissages sociaux, mais aussi à la biographie de chaque élève en tant que sujet.

Lors de nos enquêtes antérieures sur les élèves de LP (lycée professionnel) préparant un CAP ou un BEP (certificat d'aptitude professionnelle et brevet d'études professionnelles) (Jellab, 2001), nous avons privilégié une entrée empirique. Elle interroge le rapport aux contenus scolaires et professionnels, les manières d'apprendre et les lieux d'apprentissage ainsi que la légitimité et l'intérêt perçus des activités proposées.

Quatre types de rapport aux savoirs ont été identifiés : un rapport pratique aux savoirs (l'élève reste plus centré sur les apprentissages professionnels, jusqu'à surestimer les périodes de formation en milieu professionnel, au détriment des savoirs généraux, voire de l'enseignement technologique) ; un rapport réflexif aux savoirs (l'élève valorise la réflexion devant les situations pratiques en vue d'en maîtriser les enjeux ; il peut aussi manifester un intérêt particulier envers les savoirs décontextualisés, indépendamment de leurs usages, comme moyens servant l'action) ; un rapport désimpliqué aux savoirs (il s'agit de l'expérience la plus tendue de quelques élèves de LP, faiblement ou pas mobilisés sur les savoirs, avec un absentéisme récurrent et des conflits relationnels) ; un rapport intégratif-évolutif aux savoirs (la capacité de l'élève à mettre en dialogue et en cohérence différents contenus enseignés, à ne pas opposer l'enseignement général et l'enseignement technologique et professionnel).

Cette typologie caractérisant les manières de se mobiliser sur les contenus d'enseignement et de formation se retrouve chez les élèves préparant un baccalauréat professionnel, avec la prédominance d'un rapport pratique aux savoirs.

Le baccalauréat professionnel en trois ans n'a pas atténué cette tension, en dépit d'une plus forte articulation pédagogique entre l'enseignement général et l'enseignement technologique et professionnel (par exemple à travers le mode d'évaluation et l'existence d'un enseignement général lié à la spécialité). Mais plusieurs élèves interrogés ont fait l'expérience du passage d'un rapport pratique aux savoirs à un rapport réflexif ou intégratif-évolutif, au fur et à mesure du déroulement de leur scolarité. En effet, l'emprise progressive sur des contenus professionnels et la réussite, bien que parfois inégale dans l'enseignement général, favorisent l'émergence d'un intérêt pour les savoirs décontextualisés, et l'anticipation d'un avenir scolaire à moyen ou à long terme. 


\section{Le baccalauréat professionnel, un tremplin vers une ascension scolaire?}

L'alignement symbolique du baccalauréat professionnel sur les autres baccalauréats atténue le ressentiment des élèves qui, antérieurement, devaient d'abord préparer un BEP et espérer, pour $40 \%$ d'entre eux, intégrer une première professionnelle. Par ailleurs, et ce n'est pas l'un des moindres effets de la réforme du baccalauréat professionnel en trois ans, la scolarisation des contenus de formation, parce qu'elle s'inscrit davantage dans le cadre de la forme scolaire (recours à l'écrit, à la formalisation des savoirs, travail sur des notions, mobilisation de données numériques...) renforce, chez les élèves, le souhait de poursuivre des études ; il y sont encouragés par les enseignants et par la plupart des acteurs du LP qui y voient une manière de développer de nouvelles compétences, d'augmenter les chances d'insertion et de valoriser la voie professionnelle. Se dessine ainsi, chez les élèves, le projet de poursuivre des études, de vivre une ascension scolaire et de redéfinir leur identité en vivant autrement leur jeunesse.

\subsection{La signification sociologique de la poursuite d'études : une enquête qualitative auprès d'élèves de bac pro et de STS}

L'enquête menée auprès des élèves de LP ( $c f$. encadré 2) préparant un baccalauréat professionnel a révélé que sur nos vingt interlocuteurs, douze - dont six étudiants de BTS (brevet de technicien supérieur) - projetaient, dès la classe de $3^{\text {ème }}$, de poursuivre des études dans l'enseignement supérieur. Leurs propos ne traduisent certes pas une réalité objective (défaillance de la mémoire, interprétation a posteriori, etc.) ; cependant, ils manifestent, au moins partiellement, les effets d'une confrontation aux activités scolaires et professionnelles sur l'émergence du projet de poursuivre des études, donnant ainsi à la temporalité scolaire une autre configuration. Cette enquête permet d'interroger la place occupée par le baccalauréat professionnel dans la construction des trajectoires scolaires. Elle met en évidence les cohérences et les écarts entre visées institutionnelles associées à ce diplôme et manières dont les publics l'inscrivent dans des projets personnels à plus ou moins long terme. L'expérience des élèves que nous allons présenter, bien qu'elle ne soit pas représentative de tout le public ayant effectué un parcours en LP, témoigne de l'effet formateur des savoirs, en ce que leur maîtrise dessine aussi des projets d'une ascension scolaire et sociale.

On peut considérer, à l'instar des travaux de Stéphane Beaud (2002), que les bacheliers professionnels désireux de poursuivre des études cherchent à prendre une revanche sur le système scolaire, et plus particulièrement sur l'orientation subie en fin de collège qui les aurait privés d'une scolarité dans la "voie normale » que constitue le lycée général et technologique. Cette hypothèse ne correspond pas à tous les élèves préparant un baccalauréat professionnel. Dans la propension à poursuivre des études, nous observons l'effet d'un processus d'appropriation de son expérience scolaire ; en prenant appui sur la maîtrise de 
contenus professionnels et de savoirs, cette appropriation génère une adhésion à la cause scolaire, bien que celle-ci puisse être motivée par une pluralité de buts (éviter l'entrée précoce sur le marché du travail, vivre sa jeunesse selon le modèle de l'étudiant, acquérir une légitimité sociale en étant plus diplômé...).

\section{Encadré 2 : Une enquête qualitative au sein de deux lycées du Nord}

Pour se saisir des enjeux sociologiques contenus dans les aspirations à la poursuite d'études à l'issue du baccalauréat, nous avons mené une enquête qualitative auprès de vingt élèves, dont sept filles. Les élèves étaient âgés de 18 à 22 ans, douze d'entre eux étaient scolarisés en terminale bac pro, six en STS (sections de technicien supérieur) et deux en première professionnelle.

Les entretiens ont été menés entre 2013 et 2014, dans deux lycées professionnels de l'académie de Lille, dont l'un est situé au sein d'un lycée polyvalent. Cette académie se caractérise par l'importance de l'offre de formation vers la voie professionnelle, le taux d'orientation vers le LP (lycée professionnel) en fin de $3^{\text {ème }}$ étant de près de $32 \%$ pour une moyenne nationale de $26 \%$.

Sans prétendre à l'exhaustivité, les enseignements dégagés de cette enquête laissent apparaître des dynamiques socio-subjectives attestant de l'effet de la réforme du baccalauréat professionnel sur les projets d'avenir, et sur une certaine prise de distance avec l'entrée immédiate sur le marché du travail.

Démarche centrale de notre recherche, les entretiens menés avec les élèves ont été précédés par des choix sociologiquement " significatifs ». Les interlocuteurs ont en effet été choisis pour le caractère "significatif » (Michelat, 1975) et non représentatif de leur expérience. Nous avons cherché à rencontrer des élèves des deux sexes, scolarisés dans des spécialités différentes, à des étapes différentes de leur parcours et dont l'origine sociale, bien que de milieu populaire, laisse apparaître une diversité de situations familiales. Les élèves interrogés et le matériau recueilli permettent d'identifier les enjeux sociaux et subjectifs participant du rapport aux savoirs et au bac pro 3 ans. En ce sens, ils ne sauraient être représentatifs de la diversité des manières d'être aux études mais autorisent des enseignements qualitatifs que seules d'autres recherches pourraient conforter, réinterroger ou contester.

L'objectif de l'entretien non directif tel que le concevait Guy Michelat est d'être un moyen rendant compte "des systèmes de valeurs, normes, représentations, symboles propres à une culture ou à une sous-culture » (op. cit., p. 230); nous y avons davantage vu un outil permettant de mettre en dialogue les élèves avec leur expérience, dont la singularité n'exclut pas l'existence de régularités collectives.

Plutôt directifs, les entretiens étaient menés avec un fil conducteur, celui d'une centration sur la trajectoire scolaire et sociale des élèves. Axés sur l'histoire (sociale et scolaire) de l'élève, sur son expérience en LP et en dehors de lui, les entretiens étaient nourris d'éléments recueillis lors d'enquêtes précédentes, ce qui permettait, par des effets de relance, d'amener l'interlocuteur à restituer son expérience tout en l'objectivant partiellement. D'une certaine façon, et sans véritablement se confondre, l'entretien de recherche est proche de l'entretien biographique ; il révèle en effet une dynamique subjective dans laquelle s'entremêlent l'habitus et l'expérience personnelle socialement et contextuellement située. L'entretien biographique de recherche permet de penser l'expérience scolaire en interrogeant son versant subjectif face aux épreuves objectives (Martuccelli, 2006). Cette perspective méthodologique, proche de la sociologie de l'expérience, est également portée par la sociologie clinique. Le récit de vie - sollicité par le chercheur - devient alors révélation dialectique du singulier et du collectif et permet de " comprendre en quoi un individu est le produit d'une histoire dont il cherche à devenir sujet » (de Gauléjac, 1995, p. 328). 


\section{Suite encadré 2}

Nous avons veillé à ce que quelques points soient soulevés avec tous les élèves rencontrés, à savoir :

- Les classes fréquentées antérieurement et ce que l'élève y a vécu ;

- Les moments de transition (école primaire, collège, LP) ;

- Les modalités de l'orientation à l'issue du collège et du LP vers l'enseignement supérieur ;

- Le sens des matières et leur spécificité ;

- Le rapport à autrui (enseignants, camarades de classe, copains, famille...) ;

- Le sens de l'apprendre et les contextes d'apprentissage (qu'est-ce qu'apprendre ? où apprendon ? qu'apprend-on en LP ? qu'apprend-on en stage ? qui apprend et comment apprend-on ?) .

\subsection{Préparer un bac pro et souhaiter poursuivre des études supérieures : revanche ou adhésion à la cause scolaire?}

Centré sur l'effet potentiel de la réforme du bac pro 3 ans quant à la volonté de poursuivre les études dans l'enseignement supérieur, notre travail de terrain s'est attaché à repérer, chez la douzaine d'élèves exprimant ce projet ou le réalisant en STS, les dynamiques sociosubjectives susceptibles d'éclairer la hausse du nombre de bacheliers professionnels parmi la population étudiante.

Mais il serait réducteur de ne voir dans leur projet d'études que la manifestation d'un rapport instrumental et utilitaire à la formation et au diplôme, tant leur rapport à l'avenir est structuré par d'autres dimensions. Ainsi, à côté d'une émancipation par l'école, il existe d'autres mobiles, comme la participation à l'espace public favorisée par l'acquisition de nouveaux savoirs et compétences, la sociabilité juvénile que permet l'accès au statut d'étudiant, la redéfinition d'une identité sociale plus reconnue, etc. Ainsi, Bénédicte, étudiante en BTS "Assistant de manager ", vit sa scolarité sur le mode d'une ascension scolaire contribuant à redéfinir ses interactions avec ses amis, et plus particulièrement avec son copain, étudiant à la faculté de lettres. La découverte de nouvelles activités professionnelles et l'accès à des savoirs qui mettent en jeu des apprentissages relationnels mais aussi culturels l'amènent à se penser "comme une étudiante qui progresse et est capable de réussir "; elle est donc aussi légitime pour étudier dans l'enseignement supérieur que son copain, qui « est tout le temps dans ses livres».

L'histoire scolaire et familiale de chaque élève révèle plus qu'une histoire personnelle. Elle met en jeu différentes instances de socialisation et les épreuves qui les accompagnent. Ainsi, nombreux sont les élèves rencontrés à évoquer de multiples déménagements des parents, ce qui les a amenés à fréquenter différentes écoles, avec souvent des difficultés d'adaptation. D’autres font surtout état des difficultés rencontrées par les parents pour trouver un emploi, ce qui donne du marché du travail une vision peu enchantée. 
De même, les pratiques de loisirs des élèves témoignent d'un apprentissage parfois contrarié. Ainsi Aymeric, qui projetait avec ses parents de "devenir footballeur " mais qui, à l'âge de 16 ans, élève de première bac pro "Gestion et administration ", a dû " renoncer parce que les places dans le centre de formation étaient chères " et comme "en plus [il a eu] une mononucléose, ça devenait difficile d'espérer percer dans ce sport». La majorité des élèves rencontrés, à l'instar du public des lycées professionnels, proviennent de milieu populaire ; leur trajectoire ressemble bien aux tableaux peints par la littérature sociologique, à savoir que leur scolarité leur apparaît souvent comme exigeant de "faire des sacrifices ", de s'affilier à un monde aux codes culturels peu familiers (Hoggart, 1957).

Les élèves que nous avons interrogés, en dépit d'une critique récurrente du système éducatif, d'un marché du travail sélectif et augurant de difficultés d'insertion professionnelle, vivent positivement leur scolarité ou, tout au moins, y voient le moyen de réussir parce que maîtrisant autrement les exigences cognitives et les apprentissages. La réussite en LP et en STS ne s'accompagne pas d'un renoncement à soi et à sa culture sociale et familiale. Tel Rudy, 19 ans, élève de terminale bac pro "Technicien-menuisier-agenceur ", qui envisage de préparer un BTS par apprentissage. Cet élève a effectué plusieurs stages dans différentes entreprises, ce qui lui a donné " le gồt du métier » et le " désir de fonder [sa] propre entreprise ". Intéressé par le "travail manuel " mais aussi par le théâtre et le sport, Rudy observe que son parcours scolaire ne l'empêche pas "d'avoir des potes à la fois à l'université, au lycée général et en CFA ". Très conscient de l'importance des études pour " devenir quelqu'un", il espère, en étant son "propre patron", embaucher des ouvriers et des employés, et "apprendre beaucoup de choses qui nous aident dans la vie ". Il déclare : "Je dois dire que j'ai toujours eu de la chance, mes parents mont toujours laissé choisir, eux qui n'ont pas eu la chance d'aller plus loin dans les études, je me sens un peu privilégié [... A Apprendre me permet de devenir adulte, mais c'est surtout l'apprentissage de l'agencement et de la technique du travail en chantier ou en usine qui me plait le plus".

\subsection{Tableaux d'élèves}

Les cas d'élèves rapportés ici doivent être considérés comme des expériences singulières dont la lecture est susceptible de constituer un corpus informant sur les autres expériences. Ils révèlent, à leur manière, comment le projet de préparer un BTS procède de l'expérience construite en LP et lors des stages, de l'histoire biographique ainsi que du rapport aux savoirs.

Ainsi, le parcours de Fanny, élève de bac pro "Technicien en installation des systèmes énergétiques ", informe en quelque sorte sur les épreuves vécues par une élève dont le projet de poursuivre des études doit à sa position dans la fratrie et à la réussite en LP.

Le cas de Jordan, élève de première professionnelle "Accueil, relation-clients et usagers ", met en évidence une autre manière de vivre son expérience scolaire, dans laquelle il voit le moyen de s'ouvrir au monde et de s'engager tant dans un projet de poursuite d'études 
que dans la vie sociale et politique locale. Le parcours de Philippe, qui prépare un BTS "Après-vente automobile option véhicules particuliers ", montre comment l'encadrement pédagogique en LP favorise la réussite scolaire et dessine le projet de poursuivre des études dans un cadre institutionnel offrant un suivi éducatif de proximité. Il met aussi en relief les difficultés d'adaptation lors de la transition LP-STS.

Cette transition ne va pas de soi, mais peut être maîtrisée lorsque l'élève est porteur d'un projet d'émancipation permettant de vivre sa jeunesse, comme l'illustre le cas de Brahim.

Les élèves interrogés n’évoquent ni une revanche, ni le regret d'avoir été refusés pour une entrée dans la « voie normale » que constitue la seconde générale et technologique de lycée, ce qui n'exclut pas un effet de rationalisation de leur expérience. L'articulation entre une biographie singulière et un contexte de formation spécifique rend intelligible l'émergence de projets scolaires et sociaux chez les élèves de LP. Les instances de socialisation telles que l'école primaire et le collège n'agissent pas uniquement comme agents transformant des trajectoires "programmées » en destin intériorisé. Elles contribuent à structurer le regard des jeunes sur le système scolaire et génèrent un point de vue très lucide, souvent critique. Parfois, la nostalgie vis-à-vis d'une scolarité peu réussie émerge dans le propos mais assez vite, elle laisse place à un regard plus centré sur le présent et sur ce qu'il augure pour l'avenir. En effet, l'essentiel de ce que les élèves de LP appellent "le déclic » se joue dans leur affiliation aux études et le sentiment d'exercer une emprise sur leur quotidien scolaire.

Les expériences des élèves interrogés procèdent d'une mobilisation qui ne se fixe pas seulement l'emploi ou l'insertion professionnelle comme objectif princeps. Elles reposent sur l'univers symbolique des études qui apporte, en retour, une reconnaissance de ses capacités et des efforts consentis ; si l'élève peut identifier son expérience à une " revanche ", il y voit également l'occasion d'expérimenter une vie juvénile dans laquelle il neutralise l'urgence de s'insérer professionnellement.

\subsubsection{Fanny et l'émancipation scolaire par l'apprentissage du métier}

Fanny, 18 ans, est élève de terminale professionnelle «Technicien en installation des systèmes énergétiques ». Seule fille dans sa classe qui compte 24 élèves, Fanny a effectué un parcours « moyen " en école primaire, plus " compliqué » en collège. Ses résultats scolaires en classe de $3^{\text {ème }}$ lui auraient permis de "passer en seconde générale mais les profs ont dit que cétait juste ", d'autant plus que, dès la classe de $4^{\text {ème }}$, elle envisageait d'apprendre un métier dans le domaine de la climatisation. Appréciant les activités professionnelles qui demandent une mobilité et un travail sur des chantiers, cette élève a effectué un stage de découverte en classe de $3^{\text {ème }}$ : "Grâce à mon oncle qui dirige une entreprise, j'ai trouvé un patron qui m'a accueillie sur un chantier... j'ai vu travailler les ouvriers, ils posaient des radiateurs, des chauffe-eau et des systèmes de climatisation. Ça m'a beaucoup plu et les gens me disaient qu'ils allaient d'un chantier à l'autre, comme j'aime bouger, c'est ce que j'ai voulu faire en fin de $3^{\text {ime }}$ ". C'est donc bien informée sur l'un des domaines professionnels auxquels prépare le baccalauréat professionnel que Fanny s'oriente vers le LP. Ce choix ne fut pas 
facile à assumer car ses parents ne la "voyaient pas dans un métier d'homme". Seule fille d'une fratrie de quatre enfants, elle aspire à réussir ses études et à aller plus loin que «les frangins qui se sont arrêtés au CAP et qui auraient bien voulu continuer".

A l'instar de nombre d'élèves issus de milieu populaire, elle paraît portée par le projet de défier les limites qu'une histoire familiale a dû imposer à ses frères aînés : "Quand mes frères ont arrêté les études, c'était au moment où mon père était au chômage et ma mère qui gardait des enfants ne pouvait pas leur payer des études. Mon frère aîné qui a fait un CAP métallier a dû très vite bosser et même maintenant, il dit quil aurait voulu continuer [...] Si je peux, jirai en BTS, mais on m'a dit que les places sont chères. "Sa scolarité en LP s'est bien déroulée, même si elle a éprouvé certaines difficultés en sciences et en technologie. Elle s'est progressivement approprié le LP et la vie scolaire en devenant déléguée de classe (en première) et en s'investissant dans le foyer socio-éducatif. Fanny considère que les " profs de LP suivent beaucoup plus les lycéens». Elle a eu des moments de doute, voire, en fin de seconde "l'envie de tout arrêter". Ce sont les stages en entreprise et les activités professionnelles qu'elle mâ̂trise assez bien qui lui ont "redonné le goût d'apprendre et de travailler".

Fanny a validé le BEP en cours de formation et apprécie la complexité et la diversité des différentes activités préparant la certification ${ }^{4}$. Mais si sa scolarité se déroule de manière satisfaisante en LP, elle a dû faire face à des formes implicites de défiance, notamment auprès d'un enseignant en installations sanitaires et de quelques ouvriers, lors du stage en entreprise. S'il lui semble "normal " de faire l'objet de "plaisanterie par des gars de chantier», sans doute déstabilisés par le choix d'une fille d'un métier " masculin », Fanny trouve " anormal " qu'un enseignant puisse avoir un a priori négatif sur sa capacité à devenir ouvrier qualifié dans le domaine de l'installation des systèmes énergétiques : "Au début, comme jétais la seule fille de la classe, j’ai trouvé ça un peu bizarre, mais les camarades ont été sympas avec moi... C'est pas le cas d'un prof, il faisait de la techno et l'installation thermique. Il ne minterrogeait jamais, même quand je levais le bras et une fois, il m'a fait la remarque comme quoi le bac pro nétait pas fait pour les filles! Heureusement que tous les profs ne sont pas comme ça, mais ça mia choquée [...] Maintenant, ça se passe très bien et même les profs disent quils aimeraient bien que les autres élèves soient aussi motivés.»

Fanny envisage une poursuite d'études afin de préparer un BTS, et pour augmenter ses chances d'y parvenir, elle consacre "beaucoup de temps aux révisions et à la préparation des dossiers".

Le cas de Fanny atteste de la métamorphose que connaissent les élèves de LP, une métamorphose qui concerne d'abord le rapport aux savoirs et la maitrise progressive de leurs contenus comme des enjeux culturels et politiques qui les subsument (au détour d'une

\footnotetext{
4. Soulignons que les nouvelles prescriptions institutionnelles quant à la manière de préparer le baccalauréat professionnel - en l'occurrence un ancrage plus marqué du référentiel de certification dans le référentiel des activités professionnelles - semblent, dans le cas de Fanny, favoriser une cohérence entre les différents contenus d'enseignement.
} 
phrase, Fanny déclare : "Quand on étudie la mondialisation, c'est pour moi une façon de me cultiver, avec le développement des pays de l'Asie, et de la concurrence économique, je sais que les emplois en France dépendent de la qualité du travail que l'on va faire [...] Si on n'est pas compétitif, c'est notre avenir qui est fichu!»

L'expérience de Fanny manifeste un rapport intégratif-évolutif aux savoirs, davantage présent chez les filles que chez les garçons, y compris lorsqu'elles sont scolarisées dans des spécialités industrielles.

Son parcours renseigne sur les épreuves générées par un choix d'orientation dans une spécialité fortement masculinisée, sur la conscience vive de ce que la poursuite d'études peut avoir comme effet pour éviter les différentes formes de domination (de genre mais aussi au travail).

Cela rejoint les analyses sociologiques qui intègrent les catégories de classe (Grignon, 1971) et de genre (Depoilly, 2014), soulignant l'intérêt heuristique de les croiser afin d'analyser les tensions et les contradictions engendrées par l'inscription d'une histoire biographique singulière dans des rapports sociaux de domination. Comme l'avait observé Jean-Pierre Terrail, la mobilisation des filles doit à "un usage de soi particulièrement intense, suscité dans des circonstances historiques où la réussite scolaire est la seule voie de l'émancipation individuelle» (Terrail, 1997).

\subsubsection{Jordan ou quand la réussite scolaire génère un engagement " politique » et le projet de préparer un BTS}

Jordan, 19 ans, est titulaire d'un CAP «Employé de commerce multi-spécialités» (ECMS), obtenu avec 17 de moyenne. Il est actuellement en première professionnelle "Accueil, relation-clients et aux usagers " (ARCU) et est premier dans une classe de douze élèves.

Issu d'une famille qui compte huit enfants (père retraité et mère au foyer), Jordan manifeste le souci de bien réussir au LP afin d'augmenter ses chances d'insertion : "J'ai deux grandes sours qui bossent dans l'intérim, c'est pas une vie quand on ne sait jamais si on aura du travail ou pas le lendemain!» Le parcours scolaire de cet élève a été marqué par des difficultés. A l'école primaire, cela s'est "moyennement bien passé» (il y a redoublé une classe). Les difficultés ont commencé au collège où il a dû s'orienter en $3^{\text {ème }}$ d'insertion : "Je pense

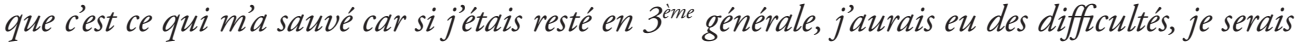
à la rue, sans rien..."

A l'issue du collège, il a obtenu le Certificat de formation générale, titre qu'il considère comme "important, ça [lui] a donné de la fierté ». Puis il s'est orienté vers le CAP ECMS : "Je l'ai choisi selon ce qu'on mia donné comme choix. Au collège, je me souviens d'une feuille jaune, je m'en souviens, il n'y avait que quelques formations, j'ai choisi ça faute de mieux, peut-être que j'aurais choisi autre chose! ». Pour lui, le CAP, "c'est génial pour une remise à niveau... ça m’a donné confiance pour aller en bac pro". En comptabilisant toutes les périodes de formation en milieu professionnel, il a à son actif plusieurs mois de stages 
(une semaine sur deux en $3^{\text {ème }}$ d'insertion pendant toute l'année, plus quatre mois cumulés lors des deux ans de CAP). Il est passé directement en première professionnelle du fait de sa bonne moyenne en CAP. Jordan pense que son choix d'orientation a été limité par l'offre locale, peu développée dans le bassin d'éducation et de formation. Concernant les enseignements en général, il déclare (et indique qu'il parle au nom des élèves puisqu'il est délégué au conseil de la vie lycéenne et au CA) : "Ici, on aime beaucoup la mise en situation, la pratique, et moins les cours. On nous apprend des choses en accueil, avec des mots, des expressions qu'on n'utilisera jamais en accueil ». Selon Jordan, ses atouts réfèrent à son "sérieux ", à son " assiduité " et ses points faibles concernent les mathématiques, qu'il n'aime guère. Son projet est de devenir adjoint administratif, hôte d'accueil, et peut-être faire de la politique. Être élu lui permettra de "défendre les petites gens", les gens comme "les élèves de LP».

Jordan pense devoir sa réussite à des enseignants qui ont su l'aider. Alors qu'il n'aime pas les mathématiques, il insiste pour dire que "la prof de maths, on peut lui poser 100 fois la même question, elle répondra 100 fois pour apporter de l'aide". Il est également élogieux à l'égard de ses parents "qui triment pour s'en sortir, et qui disent que si les enfants réussissent leurs études, c’est aussi leur réussite!". Au niveau des manières d'apprendre, il avance : "Pour apprendre, j’ai de gros problèmes de mémoire, j’essaie mais sans succès. J'ai une tablette, j'essaie de l'utiliser en anglais, en espagnol... Je trouve que c'est ridicule d'infliger de l'espagnol aux élèves... J'apprends en classe [...] Il n'y a pas de lien entre les matières, on ne va pas faire une équation du second degré en accueil! " Son implication dans la vie de l'établissement témoigne d'une bonne intégration et d'une certaine " politisation " des enjeux liés à la scolarité, puisque la réussite dans les études l'amène à se penser comme un " rescapé » devant ses meilleurs résultats à la mobilisation des enseignants.

Jordan entend poursuivre des études supérieures tout en s'engageant dans l'espace public: "Si jai mon bac avec une bonne moyenne, je me vois bien continuer, préparer un BTS par exemple. Mais j'aimerais aussi consacrer du temps à la vie sociale, j'aime aller vers les gens et les aider si je peux."

Jordan, dont la scolarité relève d'un rapport pratique aux savoirs, manifeste davantage d'intérêt pour les contenus professionnels et pour les enseignements qui interrogent la vie quotidienne.

\subsubsection{Philippe et la nécessité $d^{\prime}$ '« être encadré » pour étudier : le parcours scolaire au miroir de la réussite en STS}

Le parcours de Philippe, 19 ans, issu de milieu ouvrier, est assez classique, avec quelques difficultés scolaires rencontrées à l'école primaire et au collège, difficultés qu'il ne sépare pas de la nature des relations aux enseignants. Il est en première année de BTS «Aprèsvente automobile, option véhicules particuliers » (AVA). 
Philippe insiste, lors de l'entretien, sur le fait qu'il a besoin de l'attention des enseignants, d'un cadre scolaire plus structuré mais craint fortement l'évaluation qui le "stresse ". "A l'école primaire, j'avais un fort caractère, j'ai redoublé le CM2, parce que j'avais des difficultés en français... mais c'est le prof qui a voulu que je redouble, ça n'a servi à rien!". Elève de CM2 (cours moyen $2^{\mathrm{e}}$ année), lors d'une journée d'immersion au sein du collège de rattachement, il a décidé qu'il ne rejoindrait pas cet établissement public à la rentrée scolaire : "J'ai passé une journée avec des élèves de G'é, il y en a un qui m'a traité toute la journée, il me cherchait et m'a menacé... Alors, j'ai dit à mes parents que je préférais aller dans un collège privé. J'ai été à Notre-Dame ". Le cadre plus "strict»" du collège n'a pas empêché Philippe de "se faire remarquer", d'être puni à plusieurs reprises. Pourtant, il déclare "aimer" les enseignants exigeants et soucieux de le faire progresser. Cela confirme quelques observations effectuées auprès d'élèves de LP et montre que les exigences scolaires, doublées d'une attention bienveillante à leur égard, à leur progression, créent un espace de confiance pour apprendre. En classe de $4^{\text {ème }}$, Philippe a "pris enfin [sa] place ", au sens où il réalise qu'il est un "vrai élève ", obtenant la moyenne et se sentant plus à l'aise dans les études. Il semble qu'il ait effectué sa classe de $3^{\text {ème }}$ dans un dispositif aménagé ou adapté (il s'agit probablement d'une classe de $3^{\text {ème }}$ technologique, qui existe encore dans quelques établissements privés).

L’orientation vers le LP est " choisie ", au sens où elle apparaît cohérente avec le niveau scolaire et le projet de devenir mécanicien. Collégien, il a effectué un stage en mécanique automobile, pendant une semaine, dans le cadre du PDMF (Parcours de Découverte des Métiers et des Formations) : "... ça m’a conforté dans mon choix, je suis plutôt manuel... je connaissais quelqu'un qui aime les voitures, et un garagiste, ami de mon père, m'a pris comme stagiaire pendant une semaine ". La crainte de ne pas réussir - "j’ai toujours peur des examens, je perds mes moyens » - l'a dissuadé de trouver un maître d'apprentissage : "En entreprise, le patron va exiger un travail bien fait, pas de défaut, donc un apprenti, il risque de faire des bêtises. " L'appréhension vis-à-vis de la formation conduit Philippe à craindre d'échouer alors que, précisément, l'apprentissage est censé le préparer à la maitrise de compétences et de connaissances "pratiques». Philippe estime que "le baccalauréat professionnel en 3 ans, c'est une bonne chose parce qu'on gagne du temps" et "on progresse en même temps en allant du plus simple au plus dur».

La réussite en $\mathrm{LP}^{5}$ a conduit cet élève à vivre une métamorphose, sur fond d'une meilleure " motivation ": "J'ai été motivé parce que j’ai réussi... J'étais premier pendant 3 ans, au début, on était 30 et à la fin, on s'est retrouvé à 24. " Le " déclic " se produit au moment où Philippe réalise qu'il peut réussir, qu'il est meilleur comparé à d'autres élèves, dont certains "se sont orientés là, sans savoir pourquoi ", notamment ceux de la spécialité chaudronnerie (sous-entendant par-là, que lui, élève de bac pro "Maintenance des véhicules

5. Précisons que si tous les élèves interrogés apprécient la réforme du bac pro 3 ans, certains d'entre eux regrettent que les stages - périodes de formation en milieu professionnel - soient courts, les 22 semaines qui y sont consacrées durant les trois années leur paraissant insuffisantes. 
automobiles ", est plutôt privilégié d'être dans une spécialité sélective). Le diplôme intermédiaire n'est pas perçu comme important en soi mais comme un entraînement pour le baccalauréat professionnel : "Le BEP, c'est un diplôme qui ne m'a pas servi. Je savais que si javais le BEP, il suffirait de travailler un peu plus pour avoir le bac pro, c'est un bac pro blanc pour moi...".

Cet élève a réussi sa scolarité en LP, les relations aux enseignants étant meilleures qu'en collège. Ce sont d'ailleurs ses professeurs de mécanique qui l'ont "poussé à demander un $B T S$ ». Il a acquis une certaine confiance en soi qui l'a amené non seulement à chercher, sans succès, une entreprise en vue de préparer un BTS par apprentissage, mais aussi à intégrer un lycée polyvalent et à y être interne.

Les études en première année de BTS sont plutôt bien entamées. Le bulletin montre une moyenne de 11,2 au premier semestre, avec de meilleurs résultats en technologie (en atelier, il a une moyenne de 14,5 alors que la moyenne de la classe est de 13,5) et de bonnes appréciations en général. Il est $12^{\text {ème }}$ dans une classe qui compte trente étudiants. C'est en enseignement général qu'il éprouve des difficultés, notamment en mathématiques, avec une moyenne de 7,2, pour une moyenne de classe de 10,2 ("le prof de maths est étonné de voir que nous n'avons pas fait en bac pro le calcul des intégrales!") et en français (il a des difficultés à l'écrit).

Philippe observe une nette différence entre l'enseignement en BTS et en baccalauréat professionnel : "Au LP, on avait un cours et beaucoup d'exercices, en BTS, il y a le cours, mais on n'a qu'un ou deux exercices et après, on passe à autre chose... ". Et c'est sans doute au niveau de la transition bac pro-STS que se situe l'enjeu d'une politique scolaire ambitieuse, en particulier pour favoriser l'adaptation d'élèves qui font souvent, à l'instar de Philippe, et à l'image des cinq autres élèves rencontrés lors de cette enquête, l'expérience d'une difficile affiliation à un nouvel univers scolaire, alors que leur parcours en LP est en général assez bien réussi.

L'intérêt progressif mais récent pour la lecture de romans et d'essais montre une certaine évolution du rapport au savoir chez Philippe, oscillant entre valorisation des contenus professionnels et attrait pour des contenus culturels proches de la culture scolaire académique.

\subsubsection{Brahim ou quand les études supérieures permettent de vivre sa jeunesse}

Brahim, 21 ans, est scolarisé en deuxième année de BTS « Après-vente automobile, option véhicule particuliers » (AVA). Issu de milieu ouvrier (père ouvrier dans l'industrie, mère au foyer), il a effectué un parcours scolaire marqué par des difficultés à l'école primaire : " $A$ l'école primaire, ce n'était pas très bon, ce qui était difficile, c'était l'orthographe, j'ai dû redoubler le CE1, javais de gros problèmes en lecture... ". Il s'est heurté à de grandes difficultés au collège où "ce nétait pas évident" et ce, jusqu'en fin de $4^{\text {ème. }}$. Au contact d'un enseignant de français en $4^{\text {ème }}$, il a eu « le déclic », car il lui a donné l'envie de réussir. 
Il a ainsi pu mieux réussir sa classe de $3^{\text {ème }}$ technologique. L'enseignement technologique et ses différents contenus et supports, la pratique en atelier comme les stages en entreprise lui ont assuré une meilleure maîtrise de sa scolarité et, " dès la classe de seconde, [il a] envisagé de faire un BTS».

Il considère que les enseignants "suivent bien les élèves " et que c'est à ce titre qu'il a obtenu son baccalauréat professionnel avec 15 de moyenne. Issu d'une famille d'origine algérienne et d'une fratrie de cinq enfants, cet élève insiste sur la place que l'école revêt aux yeux de ses parents : "Pour mes parents, on ne peut s'en sortir que par l'école... Ma mère a toujours été derrière nous, pour les devoirs, pour aller voir les profs quand ça n'allait pas bien [...] La fierté de mes parents, c'est quand on revient à la maison avec de bonnes notes, avec des diplômes, comme ma grande sour qui est professeure des écoles...".

Il a choisi de poursuivre ses études en BTS car il est passionné par le diagnostic et la réparation de véhicules. Sa scolarité en STS est jalonnée par des difficultés dans l'enseignement général, sur fond de changement au plan des exigences scolaires. Ainsi, il observe qu'en français, il lui faut maîtriser des compétences en synthèse et en expression personnelle, alors qu'en baccalauréat professionnel, il lui « suffisait » de lire des textes et de répondre à des questions.

Il a fallu qu'il passe " un cap ", celui des premiers mois en première année, qui ont vu certains élèves décrocher. Pour Brahim, le fait de réussir dans les enseignements technologiques et lors de la pratique en atelier a eu un effet motivant et lui a permis de persévérer. Le rapport pratique aux savoirs, prédominant chez cet étudiant, n'empêche pas une relative mobilisation sur l'enseignement général, même si celle-ci procède de la nécessité et moins de l'intérêt pour les activités intellectuelles.

Les quatre portraits d'élèves présentés dans le cadre de cet article illustrent des expériences constituant une grille de lecture permettant d'apprécier les effets de la réforme bac pro 3 ans sur le rapport aux savoirs et sur l'élaboration des projets d'avenir. Ces projets ne procèdent pas des seules variables scolaires - par exemple le niveau scolaire autorisant ou non la poursuite d'études -, ni de la seule opportunité offerte par un accueil massif et direct en bac pro, et en STS pour les bacheliers professionnels. Les projets relèvent également de dynamiques sociales (place et statut des diplômes au moment où la classe ouvrière se recompose et devient moins visible) et familiales (rapports intergénérationnels, construction d'une identité sociale étudiante plus reconnue ou légitime...).

La propension à la poursuite d'études dans l'enseignement supérieur s'est affirmée avec la généralisation du bac pro 3 ans et dessine un mouvement qui devrait se renforcer. Cela tient à l'accroissement très significatif du nombre de bacheliers professionnels, qui passe de 119000 en 2010 à 190000 en 2014. " $42 \%$ des lauréats poursuivent leurs études à l'issue du baccalauréat. Ils sinscrivent très majoritairement en sections de techniciens supérieurs, où ils obtiennent des résultats mitigés : près de la moitié d'entre eux obtiennent leur diplôme. Lorsqu'ils rejoignent les formations générales à l'université, ils sont très peu nombreux 
à y obtenir leur licence" (Péan, 2012). Ce taux de poursuite d'études couvre en réalité des situations bien hétérogènes : ainsi, et si l'on excepte les apprentis et ceux qui sont en contrat de professionnalisation, à peine un peu plus d'un bachelier sur quatre poursuit des études en formation initiale, en STS et à l'université en l'occurrence ${ }^{6}$. Ce taux est néanmoins en forte progression puisqu'il était de $17 \%$ en 2000, de $23 \%$ en 2005 et approche les $27 \%$ en 2012.

\section{Conclusion}

La mise en place d'un baccalauréat préparé en 3 ans après la classe de $3^{\text {ème }}$ a eu un effet d'appel sur une partie des élèves qui, tout en aspirant à poursuivre des études supérieures, considèrent que le lycée professionnel (LP) leur assure un " compromis » entre l'école et le marché du travail. Au moment où le baccalauréat professionnel enregistre une augmentation de ses effectifs, le baccalauréat technologique connaît une baisse de sa population (entre 2009 et 2011, parmi l'ensemble des effectifs de terminale, la part des élèves scolarisés en STG - sciences et technologies de la gestion, et en STI - sciences et technologies industrielles ${ }^{7}$ - passe respectivement de 13,2 et 6,1 à 11,7 et 5,4\%).

Les différents portraits d'élèves rapportés font écho à ceux de leurs camarades qui ont envisagé la voie professionnelle comme une étape de leur parcours scolaire, et non comme propédeutique à l'entrée sur le marché du travail. En 2013, 36 \% des bacheliers professionnels souhaitant poursuivre leurs études ont été acceptés en BTS et le taux de réussite à ce diplôme avoisine aujourd'hui les $55 \%$. Ainsi, au bout d'une trentaine d'années d'existence, le baccalauréat professionnel a vu évoluer ses missions, et redéfinir les priorités qui lui sont assignées : de diplôme censé former des futurs ouvriers et employés qualifiés pour alimenter prioritairement le marché du travail, la poursuite d'études étant une possibilité offerte à une minorité, c'est plutôt la tendance inverse qui tend à s'affirmer aujourd'hui.

L'enquête évoquée plus haut, menée par P-Y. Bernard et V. Troger (2011), confirme désormais qu'une majorité des élèves entrant en seconde professionnelle envisage de poursuivre des études à l'issue du baccalauréat. Dans le même temps, et depuis 2011, on enregistre un accroissement de la part des bacheliers professionnels parmi l'ensemble des bacheliers et, pour la première fois, le nombre de diplômés dépasse celui des bacheliers technologiques (27,7\% contre 23,6 \%). Mais la propension à la poursuite d'études, pour contribuer à affaiblir la finalité initiale fixée au baccalauréat profes-

6. La proportion des bacheliers professionnels poursuivant leurs études dans l'enseignement supérieur reste nettement inférieure à celle des bacheliers généraux et technologiques : en 2012, celle-ci était respectivement de 98 et de $76 \%$.

7. Depuis 2012, ces deux baccalauréats ont changé de nom : STG est devenu STMG (sciences et technologies du management et de la gestion) et STI est remplacé par le bac STI2D (sciences et technologies de l'industrie et du développement durable). 
sionnel, à savoir l'accès à l'emploi ouvrier ou employé qualifié, pour signifier aussi l'attractivité d'un diplôme désormais aligné sur les autres baccalauréats puisque préparé en 3 ans, reste concomitante à des paradoxes que l'on peut observer auprès des élèves. Ainsi, leur volonté de poursuivre des études ne lève pas leur réticence à l'égard de la forme scolaire et des savoirs perçus comme théoriques et parfois désincarnés. Dans le même temps, et conscients de ce que le diplôme peut constituer pour affronter les contraintes du marché du travail, ces élèves dont l'origine sociale est majoritairement populaire, voient dans la poursuivre des études l'opportunité de faire valoir leur réussite et d'exister socialement sur le mode d'une ascension davantage scolaire que sociale (Jellab, 2014). Nombreux sont les élèves à faire état de l'expérience de l'un des membres de leur fratrie quant au difficile accès au marché du travail mais aussi à la poursuite d'études quand, auparavant, à l'issue d'un CAP ou d'un BEP, il fallait passer le filtre de la sélection amenant au bac pro. Si les élèves rencontrés semblent, pour une majorité d'entre eux, avoir assumé leur orientation vers le LP, c'est parce que le bac pro 3 ans ne leur apparait plus comme l'horizon d'un cheminement scolaire ultime mais comme un diplôme ouvrant sur un champ de possibles, comme la poursuite des études, selon différents statuts - en formation initiale scolaire ou sous statut d'apprenti -, le fait de vivre sa jeunesse sur le mode de l'expérience étudiante et la possibilité de disposer d'un temps plus long afin de construire et de consolider un projet professionnel. Ainsi, et c'est sans doute l'un des autres effets de la réforme bac pro 3 ans, les élèves rencontrés sont peu enthousiasmés par la persptective d'entrer sur le marché du travail à l'obtention du bac, moins parce qu'ils craignent d'occuper un statut professionnel dominé ou précaire que parce que, plus jeunes que les anciennes générations, ils aspirent à poursuivre de manière logique leurs études, sans minorer les effets de cet allongement sur leur insertion professionnelle future. Pour autant, la diversification des publics scolaires, le prestige inégal des spécialités sur le marché du travail, les exigences contenues dans les formations post-baccalauréat dessinent les aléas de la réforme du bac pro, puisqu'ils augurent de devenirs sociaux et professionnels différenciés, que seule une analyse longitudinale est à même de mettre au jour. Par ailleurs, la désaffection d'une frange de plus en plus importante d'élèves vis-à-vis du marché du travail, au profit de la poursuite des études, pourrait paradoxalement renforcer la disqualification sociale et professionnelle des bacheliers, et notamment de ceux qui préfèrent entrer directement dans la vie active. En effet, l'élévation des niveaux de qualification des sortants favorise des processus d'exclusion et de précarisation des moins diplômés, même si les usages économiques des diplômes restent soumis à des politiques salariales, variables selon les branches et les stratégies des employeurs (Moreau \& al. 2002). 


\section{- Bibliographie}

Beaud S. (2002), $80 \%$ au bac et après? Les enfants de la démocratisation scolaire, Paris, La Découverte.

Beaud S. , Pialoux M. (2003), Retour sur la condition ouvrière, Paris, Fayard.

Bernard P.-Y., Troger V. (2011), Le baccalauréat professionnel en trois ans : perspectives et risques pour les lycéens, Rapport de recherche, DEPP.

Charlot B. (1987), L'école en mutation, Paris, Payot.

Charlot B. (1997), Du rapport au savoir. Eléments pour une théorie, Paris, Anthropos.

Charlot B., Emin L., Jellab A. (2002), L'abandon scolaire en cours de formation : le cas des élèves de BEP, Paris, université Paris8/MEN-DESCO.

Chauvel L. (2011), Le destin des générations, Paris, PUF.

Depoilly S. (2014), Filles et garçons au lycée pro, Rennes, PUR.

Doriath B., Cuisinier J.-F. (2009), La rénovation de la voie professionnelle, rapport IGEN et IGAEN, nº 2009-065 au Ministre de l'Éducation nationale, Paris, ministère de l'Éducation nationale.

Eckert H. (1999), « L'émergence d'un ouvrier bachelier », Revue française de sociologie, Vol. 40, nº.

Gauléjac V (de). (1995), "Le récit de vie, entre la psychanalyse et la sociologie clinique ", Lorientation scolaire et professionnelle, $\mathrm{n}^{\circ} 3$.

Grignon C., (1971), L'ordre des choses, Paris, Minuit.

Hoggart R. (1957), La culture du pauvre, Paris, Minuit.

Ilardi V., Sulzer E., (2013), «Entrer sur le marché du travail à l'issue de baccalauréat professionnel ", Le Baccalauréat professionnel : insertion et/ou poursuite d'études ?, CPC Info, 53.

Jellab A. (2001), Scolarité et rapport aux savoirs en lycée professionnel, Paris, PUF.

Jellab A. (2003), « Entre socialisation et apprentissages : les élèves de lycée professionnel à l'épreuve des savoirs ", Revue française de pédagogie, nº 142.

Jellab A. (2005, a), « Les enseignants de lycée professionnel et leurs pratiques pédagogiques : entre lutte contre l'échec scolaire et mobilisation des élèves ", Revue française de sociologie, Vol. 42, $\mathrm{n}^{\circ} 2$.

Jellab A. $(2005$, b), "Le travail enseignant en lycée professionnel et ses paradoxes ", Sociologie du travail, $47-4$. 
Jellab A.(2009), Sociologie du lycée professionnel. L'expérience des élèves et des enseignants dans une institution en mutation, Toulouse, Presses universitaires du Mirail.

Jellab A. (2014), L'émancipation scolaire. Pour un lycée professionnel de la réussite, Toulouse, Presses universitaires du Mirail.

Martuccelli D. (2006), Forgé par l'épreuve. L'individu dans la France contemporaine, Paris, A. Colin.

Michelat G. (1975), "Sur l'utilisation de l'entretien non directif en sociologie ", Revue française de sociologie, 16-2.

Moreau G. (coord.) (2002), Les patrons, l'Etat et la formation des jeunes, Paris, La Dispute.

Péan S. (2012), "Les bacheliers professionnels dans l'enseignement supérieur », Note d’information, MEN, juin 2012-04.

Ministère de l'Education nationale (2014), Repères et références statistiques.

Terrail J.-P. (1997), "La supériorité scolaire des filles », in La scolarisation de la France, (sous la direction de Jean-Pierre Terrail), Paris, La Dispute. 